\title{
A free boundary problem modeling a foam drainage
}

\author{
Thierry Colin and Pierre Fabrie,
}

Mathématiques Appliquées de Bordeaux, Université Bordeaux 1 and CNRS ESA 5466, 351 cours de la libération, 33405 Talence cedex, France.

Abstract: We study a reaction-diffusion problem with a free boundary governing the evolution of a foam. We show that the problem is globaly well-posed and that the solution converges, when the viscosity tends to zero to the solution of an initial-boundary value problem for Burgers equation.

Key words: Free boundary problem, diffusion-reaction, Burgers equation. AMS subject classification: $35 \mathrm{~K} 60,35 \mathrm{R} 35,76 \mathrm{~B} 45$.

\section{Introduction}

In this paper, we study the equation governing the evolution of a foam. A foam is a complex porous medium which consists in a set of gaz bubbles which faces are liquid films that contain only few water. These liquid films can only exist thanks to the capilary forces. Almost all the water is contained in the edges of the lattice which are called the Plateau borders. In laboratory experiments, one wants to describe how water drain into the Plateau borders when it is submitted to gravity forces in order to understand the stability properties of the foam, which has many industrial applications (see [6], [3]). One describes the foam by the evolution of the area of the Plateau borders. In the experimental procedure, the foam is contained in a vertical column and one is able to measure the area $\tilde{u}(t, x)$ of the Plateau borders at height $x \in[0, H]$ and at time $t$ (where $H$ is the height of the column) thanks to electric conductivity techniques [2]. Denote by $u(t, x)$ the dimensionless cross section area of Plateau borders at time $t$ and level $x$. The function $u(t, x)$ obeys the following equation $[6],[2]:$

$$
\left.\partial_{t} u+\partial_{x}\left(u^{2}-\varepsilon \sqrt{u} \partial_{x} u\right)=0, x \in\right] 0,1[
$$

where $\varepsilon$ is given by

$$
\varepsilon=\frac{\gamma C}{2 \pi \rho g \sqrt{\pi A_{i}}}
$$

Here $\gamma$ is the coefficient of surface tension, $C=\sqrt{\sqrt{3}-\pi / 2}$ is a geometrical constant corresponding to the form of the foam, $\rho$ is the density of water, $g$ is the acceleration of gravity and $A_{i}$ is a typical value of the area of the Plateau borders (for example, a mean value of the initial data). The number $\varepsilon$ is typically $10^{-2}-10^{-4}$ see [2] for details.

The equation (1.1) has to be completed with boundary conditions. In the case where the foam is stable during the draining, i.e. does not break, one take $u^{2}-\varepsilon \sqrt{u} \partial_{x} u=0$ at $x=0$, that means that no water enters the foam at the top level. At the bottom, the boundary that one has to impose is not clear physically. Therefore we will consider the problem posed on the half line. Equation (1.1) associated to the above boundary condition is rather classical ; we do not present any mathematical study of it, see however [2] for experimental and numerical results. Here we are interested in the case where the foam breaks during the drying process. At time $t$, the foam is confined in the domain $[\xi(t),+\infty[$, where $\xi(t)$ denotes the position of the breaking front. Physically, the foam breaks when the area of the Plateau borders reaches a critical value $\alpha>0$. Therefore, the boundary condition at $x=\xi(t)$ is $u(t, \xi(t))=\alpha$. On the other hand, conservation of quantity of water at the breaking front leads to

$$
\dot{\xi}(t)=\alpha-\frac{\varepsilon}{\sqrt{\alpha}} \partial_{x} u(t, \xi(t)),
$$


(see [2]), where $\dot{\xi}(t)$ denotes the time derivative of $\xi$. The problem we have to deal with reads

$$
\begin{gathered}
\left.\partial_{t} u+\partial_{x} u^{2}-\varepsilon \partial_{x}\left(\sqrt{u} \partial_{x} u\right)=0 \quad x \in\right] \xi(t),+\infty[ \\
u(t, \xi(t))=\alpha \\
\dot{\xi}(t)=\alpha-\frac{\varepsilon}{\sqrt{\alpha}} \partial_{x} u(t, \xi(t)) \\
\left.u(0, x)=u_{0}(x) \quad x \in\right] \xi(0),+\infty[,
\end{gathered}
$$

where $0<\alpha<1$ and $\left.u_{0}(x) \geq \alpha, \forall x \in\right] \xi(0),+\infty[$.

In laboratory experiments, one observes that the front velocity is constant. Moreover, after a time during which nothing appears, the solution decreases as $C / t$ and then stabilizes at the value $\alpha$ before the breaking front arrives. This is roughly speaking described by the entropy solution of Burgers equation

$$
\begin{gathered}
\left.\partial_{t} u+\partial_{x} u^{2}=0, \quad x \in\right] \alpha t,+\infty[ \\
u(t, \alpha t)=\alpha \\
u(0, x)=u_{0}(x)
\end{gathered}
$$

which is

$$
\begin{array}{rcrl}
u(t, x)=\alpha & \text { if } & \alpha t \leq x \leq 2 \alpha t \\
u(t, x)=\frac{x}{2 t} & \text { if } & 2 \alpha t \leq x \leq 2 u_{0}(0) t \\
u(t, x) & \text { given by } u_{0} \text { if } & & x \geq 2 u_{0}(0) t .
\end{array}
$$

Note that some discontinuity are observed experimentaly when the initial condition $u_{0}$ is not non-decreasing [2]. The hypothesis $u_{0}(x) \geq \alpha$ ensures that the solution to (1.3) propagates at velocity at least $2 \alpha$ which is strictly larger than the velocity of the free boundary. This fact will be used in last section of this paper in order to ensure the uniqueness of the entropy solution of (1.3).

In order to study (1.2) we perform the change of unknown $v(t, x)=u(t, x+\xi(t))$. Then, the equation satisfied by $v$ is

$$
\begin{gathered}
\left.\partial_{t} v-\dot{\xi}(t) \partial_{x} v+\partial_{x} v^{2}-\varepsilon \partial_{x}\left(\sqrt{v} \partial_{x} v\right)=0, \quad x \in\right] 0,+\infty[ \\
v(t, 0)=\alpha, \\
\dot{\xi}(t)=\alpha-\frac{\varepsilon}{\sqrt{\alpha}} \partial_{x} v(t, 0), \xi(0)=0 \\
v(0, x)=u_{0}(x) .
\end{gathered}
$$

In the sequel, we still denote by $u$ the solution of (1.4). The aim of this paper is to prove a global existence result for (1.2) (Theorem 2.1) and to perform the limit $\varepsilon \rightarrow 0$ in order to show that the solution converges to the solution to (1.3) (Theorem 3.1). We use energy methods and maximum principles adapted to this free boundary problem. For vanishing viscosity limit without free boundary see for example [1] or [4].

- Notations: For $v \in L^{2}(I),|v|_{2}$ denotes the $L^{2}$ norm of $v:|v|_{2}=\left(\int_{I} v^{2}\right)^{\frac{1}{2}}$, and $|v|_{\infty}$ denotes the $L^{\infty}$ norm of $v$. 


\section{Construction of strong global solutions for fixed $\varepsilon$}

In order to construct global strong solutions to (1.4), we begin with the case of a bounded interval $] 0, L[$ with homogeneous Neuman boundary condition at $x=L$. We find estimates independent of $L$ and then perform the limit when $L \rightarrow+\infty$. Note that the problem with $L<+\infty$ is interesting in itself, since it is used for numerical simulations in [2]. We therefore include it in theorem 2.1.

The aim of this section is therefore to construct global solutions for

$$
\begin{gathered}
\left.\partial_{t} u-\dot{\xi}(t) \partial_{x} u+\partial_{x}\left(u^{2}-\varepsilon \sqrt{u} \partial_{x} u\right)=0, t>0, x \in\right] 0, L[ \\
\left.u(0, x)=u_{0}(x), x \in\right] 0, L[ \\
u(t, 0)=\alpha, \partial_{x} u(t, L)=0, t \geq 0, \\
\dot{\xi}(t)=\alpha-\frac{\varepsilon}{\sqrt{\alpha}} \partial_{x} u(t, 0), t \geq 0,
\end{gathered}
$$

The hypotheses on the data $u_{0}$ and $\alpha$ are the following :

$$
\begin{gathered}
0<\alpha<1, \\
u_{0} \in W^{1, \infty}(] 0, L[), u_{0}(0)=\alpha, \\
\left.u_{0}(x) \geq \alpha, \forall x \in\right] 0, L[.
\end{gathered}
$$

We will prove

\section{Theorem 2.1}

1. - Let $u_{0}$ and $\alpha$ satisfy (2.5). There exists a unique $u \in \mathcal{C}\left(\mathbb{R}_{+} ; H^{1}(] 0, L[)\right) \cap L_{\text {loc }}^{2}\left(\mathbb{R}_{+} ; H^{2}(] 0, L[)\right), \dot{\xi} \in$ $L^{\infty}\left(\mathbb{R}_{+}\right)$satisfying (2.1)-(2.4).

Moreover,

$$
\begin{gathered}
\left.\alpha \leq u(t, x) \leq \max _{x \in] 0, L[} u_{0}(x), \forall t \geq 0, \forall x \in\right] 0, L[ \\
\alpha-\frac{\varepsilon}{\sqrt{\alpha}} \frac{1}{\left(\frac{1}{\max \partial_{x} u_{0}}+2 t\right)} \leq \dot{\xi}(t) \leq \alpha, \text { and } \partial_{x} u(t, x) \leq \frac{1}{\left(\frac{1}{\max \partial_{x} u_{0}}+2 t\right)} .
\end{gathered}
$$

- If $\partial_{x} u_{0} \geq 0$, then $\left.\partial_{x} u(t, x) \geq 0 \quad \forall t \geq 0, \quad \forall x \in\right] 0, L[$.

If $u_{0} \in H^{3}(] 0, L[)$ and satisfies besides the compatibility condition

$$
-\dot{\xi}(0) \partial_{x} u_{0}(0)+\partial_{x}\left(u_{0}^{2}-\varepsilon \sqrt{u_{0}} \partial_{x} u_{0}\right)(x=0)=0,
$$

then

$$
u \in \mathcal{C}\left(\mathbb{R}_{+} ; H^{3}(] 0, L[)\right) \cap L_{l o c}^{2}\left(\mathbb{R}_{+} ; H^{4}(] 0, L[)\right) \text { and } \xi \in W_{l o c}^{2,4}\left(\mathbb{R}_{+}\right) .
$$

2. The previous results hold in the case where $L=+\infty$ in the following sense:

- If $u_{0}-1 \in H^{1}\left(\mathbb{R}_{+}\right) \cap W^{1, \infty}\left(\mathbb{R}_{+}\right)$, then

$$
u-1 \in \mathcal{C}\left(\mathbb{R}_{+} ; H^{1}\left(\mathbb{R}_{+}\right)\right) \cap L_{\text {loc }}^{2}\left(\mathbb{R}_{+} ; H^{2}\left(\mathbb{R}_{+}\right)\right)
$$

- If $u_{0}-1 \in H^{3}\left(\mathbb{R}_{+}\right)$, then

$$
u-1 \in \mathcal{C}\left(\mathbb{R}_{+} ; H^{3}\left(\mathbb{R}_{+}\right)\right) \cap L_{\text {loc }}^{2}\left(\mathbb{R}_{+} ; H^{4}\left(\mathbb{R}_{+}\right)\right)
$$

The scheme of the proof is the following one : first we consider a regularization of the square root and construct local solutions (section 2.1). In a second step, we establish some $L^{\infty}$ bounds on $u$ and $\partial_{x} u$ thanks to maximum principle techniques and an Oleinik's entropylike estimate (section 2.2). We conclude the proof performing standard energy estimates using these uniform bounds (section 2.3). 


\subsection{Local solutions for a regularized problem.}

For $u \in \mathbb{R}$ we set

$$
f_{\eta}(u)=\min \left(\sqrt{\eta+|u|}, \sqrt{\eta+\max \left(\max _{x \in] 0, L[} u_{0}(x), \alpha\right)}\right)
$$

and we consider the following approximation of (2.1)-(2.4) :

$$
\begin{gathered}
\left.\partial_{t} u_{\eta}-\dot{\xi}_{\eta}(t) \partial_{x} u_{\eta}+\partial_{x} u_{\eta}^{2}-\varepsilon \partial_{x}\left(f_{\eta}\left(u_{\eta}\right) \partial_{x} u_{\eta}\right)=0, \quad \forall t>0, \quad \forall x \in\right] 0, L[, \\
\left.u_{\eta}(0, x)=u_{0}(x) \quad \forall x \in\right] 0, L[, \\
u_{\eta}(t, 0)=\alpha, \quad \partial_{x} u_{\eta}(t, L)=0 \quad \forall t>0, \\
\dot{\xi}_{\eta}(t)=\alpha-\frac{\varepsilon}{\sqrt{\alpha}} \partial_{x} u_{\eta} \quad \forall t>0,
\end{gathered}
$$

In the sequel, we suppose without loss of generality that $L \geq 1$, and we take $\varphi \in \mathcal{C}^{\infty}([0, L])$ such that

$$
\varphi(x) \equiv \alpha \text { for } x \leq \frac{1}{2}, \varphi(x) \equiv 1 \text { for } x \geq \frac{3}{4}, \varphi \text { non-decreasing. }
$$

Moreover, we set $v_{\eta}(t, x)=u_{\eta}(t, x)-\varphi(x)$, so that $v_{\eta}$ satisfies homogeneous boundary conditions at $x=0$ and $x=L$.

The equations satisfied by $v_{\eta}$ read as follows

$$
\begin{gathered}
\partial_{t} v_{\eta}-\dot{\xi}_{\eta}(t) \partial_{x} v_{\eta}+\partial_{x} v_{\eta}^{2}+2 \partial_{x}\left(v_{\eta} \varphi\right)-\varepsilon \partial_{x}\left(f_{\eta}\left(v_{\eta}+\varphi\right) \partial_{x} v_{\eta}\right) \\
\left.=\dot{\xi}_{\eta}(t) \partial_{x} \varphi-\partial_{x} \varphi^{2}+\varepsilon \partial_{x}\left(f_{\eta}\left(v_{\eta}+\varphi\right) \partial_{x} \varphi\right) \quad \forall t>0, \quad \forall x \in\right] 0, L[, \\
\left.v_{\eta}(0, x)=u_{0}(x)-\varphi(x), \quad \forall x \in\right] 0, L[, \\
v_{\eta}(t, 0)=0, \quad \partial_{x} v_{\eta}(t, L)=0, \quad \forall t>0 \\
\dot{\xi}_{\eta}(t)=\alpha-\frac{\varepsilon}{\sqrt{\alpha}} \partial_{x} v_{\eta}(t, 0), \quad \forall t>0 .
\end{gathered}
$$

To construct a solution to (2.10)-(2.13), we use a Galerkin approximation built on the basis of the eigenfunctions of $-\partial_{x x}^{2}$ endowed with the boundary condition (2.12). As usual, it is enough to find some a priori estimates on $v_{\eta}$.

Let us note

$$
f_{\text {min }}^{\eta}=\min _{x \in] 0, L[} f_{\eta}\left(v_{\eta}(t, x)+\varphi(x)\right)
$$

and

$$
f_{\max }^{\eta}=\max _{x \in] 0, L[} f_{\eta}\left(v_{\eta}(t, x)+\varphi(x)\right) .
$$

We remark that $f_{\text {min }}^{\eta}(t) \geq \sqrt{\eta}$ and $f_{\text {max }}^{\eta} \leq \sqrt{\eta+\max \left(\max _{x \in] 0, L[} u_{0}(x), \alpha\right)}$ for any $t \geq 0$.

- $L^{2}$ estimate : Multiplying (2.10) by $v_{\eta}$ and integrating over $] 0, L[$ leads to

$$
\begin{aligned}
& \frac{1}{2} \frac{d}{d t}\left|v_{\eta}\right|_{2}^{2}+\int_{] 0, L[} \partial_{x} \varphi(x) v_{\eta}^{2}(x)+v_{\eta}(t, L)^{2}+\eta f_{\text {min }}^{\eta}(t)\left|\partial_{x} v_{\eta}\right|_{2}^{2} \\
& \leq \frac{1}{2}\left|\dot{\xi}_{\eta}(t)\right| v_{\eta}(t, L)^{2}+\frac{2}{3}\left|v_{\eta}(t, L)\right|^{3} \\
& +\left|\dot{\xi}_{\eta}(t)\right|\left|\partial_{x} \varphi\right|_{2}\left|v_{\eta}\right|_{2}+\left|\partial_{x}\left(\varphi^{2}\right)\right|_{2}\left|v_{\eta}\right|_{2}+\varepsilon f_{\text {max }}^{\eta}\left|\partial_{x} \varphi\right|_{2}\left|\partial_{x} v_{\eta}\right|_{2} .
\end{aligned}
$$

Now, recall that for all $\psi \in H^{1}(] 0, L[)$ such that $\psi(0)=0$ (or $\psi(L)=0$ ) one has

$$
|\psi|_{\infty} \leq \sqrt{2}|\psi|_{2}^{1 / 2}\left|\partial_{x} \psi\right|_{2}^{1 / 2}
$$


Using (2.15) and Young's inequality in (2.14) leads to

$$
\frac{d}{d t}\left|v_{\eta}\right|_{2}^{2}+\varepsilon f_{\min }^{\eta}\left|\partial_{x} v_{\eta}\right|_{2}^{2} \leq C_{\varepsilon, \eta}\left\{\left|\dot{\xi}_{\eta}(t)\right|^{2}\left|v_{\eta}\right|_{2}^{2}+\left|v_{\eta}\right|_{2}^{6}+1\right\}
$$

where $C_{\varepsilon, \eta}$ depends on $\varepsilon, f_{\min }^{\eta}, f_{\max }^{\eta}$ but not on $L$.

Of course in order to control $\dot{\xi}_{\eta}$ one needs a bound on $\partial_{x x}^{2} v_{\eta}$ in $L^{2}(] 0, L[)$.

- $H^{1}$ estimates : Multiplying (2.10) by $-\partial_{x x}^{2} v_{\eta}$ and integrating over $] 0, L[$ leads to

$$
\begin{aligned}
& \frac{1}{2} \frac{d}{d t}\left|\partial_{x} v_{\eta}\right|_{2}^{2}-\frac{1}{2} \dot{\xi}_{\eta}(t)\left(\partial_{x} v_{\eta}(t, 0)\right)^{2}+\int_{] 0, L[}\left(\partial_{x} v_{\eta}\right)^{3}+\alpha\left(\partial_{x} v_{\eta}(t, 0)\right)^{2} \\
& +3 \int_{] 0, L[}\left(\partial_{x} v_{\eta}\right)^{2} \partial_{x} \varphi+2 \int_{] 0, L[} \partial_{x x}^{2} \varphi v_{\eta} \partial_{x} v_{\eta} \\
& +\varepsilon f_{\min }^{\eta}\left|\partial_{x x}^{2} v_{\eta}\right|_{2}^{2}+\varepsilon \int_{] 0, L[} f_{\eta}^{\prime}\left(v_{\eta}+\varphi\right)\left(\partial_{x} v_{\eta}+\partial_{x} \varphi\right) \partial_{x} v_{\eta} \partial_{x x}^{2} v_{\eta} \\
& \leq\left|\dot{\xi}_{\eta}\right|\left|\partial_{x} \varphi\right|_{2}\left|\partial_{x x}^{2} v_{\eta}\right|_{2}+\left|\partial_{x x}^{2} \varphi\right|_{2}\left|\partial_{x x}^{2} v_{\eta}\right|_{2} \\
& +\varepsilon f_{\max }^{\eta}\left|\partial_{x x}^{2} \varphi\right|_{2}\left|\partial_{x x}^{2} v_{\eta}\right|_{2}+\varepsilon f_{\text {max }}^{\eta \prime}\left|\partial_{x} v_{\eta}\right|_{2}\left|\partial_{x x}^{2} v_{\eta}\right|_{2} .
\end{aligned}
$$

Using again (2.15) and Young's inequality, we get

$$
\frac{d}{d t}\left|\partial_{x} v_{\eta}\right|_{2}^{2}+\varepsilon f_{m i n}^{\eta}\left|\partial_{x x}^{2} v_{\eta}\right|_{2}^{2} \leq C_{\varepsilon, \eta}\left(\left|\dot{\xi}_{\eta}\right|^{2}\left(1+\left|\partial_{x} v_{\eta}\right|_{2}^{2}\right)+\left(1+\left|\partial_{x} v_{\eta}\right|_{2}^{6}\right)\right)
$$

where the term $\varepsilon \int_{] 0, L[} f_{\eta}^{\prime}\left(v_{\eta}+\varphi\right)\left(\partial_{x} v_{\eta}\right)^{2} \partial_{x x}^{2} v_{\eta}$ has been controled by

$$
C \varepsilon f_{\text {max }}^{\eta^{\prime}}\left|\partial_{x} v_{\eta}\right|_{\infty}\left|\partial_{x} v_{\eta}\right|_{2}\left|\partial_{x x}^{2} v_{\eta}\right|_{2} \leq C \varepsilon\left|\partial_{x} v_{\eta}\right|_{2}^{3 / 2}\left|\partial_{x x}^{2} v_{\eta}\right|_{2}^{3 / 2} .
$$

Once again, $C_{\varepsilon, \eta}$ depends only on $\varepsilon, f_{\min }^{\eta}, f_{\max }^{\eta}, f_{\max }^{\eta \prime}$. The dependence of the right-handside of $(2.17)$ on $\xi_{\eta}$ contains only $\left|\dot{\xi}_{\eta}\right|$ as in the energy estimate (2.16). Therefore, since $\dot{\xi}_{\eta}$ is expressed in terms of $\partial_{x} v_{\eta}(t, 0)$, we conclude as follows: thanks to (2.15), on has

$$
\left|\dot{\xi}_{\eta}(t)\right| \leq C\left(1+\left|\partial_{x} v_{\eta}\right|_{\infty}\right) \leq C\left(1+\left|\partial_{x} v_{\eta}\right|_{2}^{1 / 2}\left|\partial_{x x}^{2} v_{\eta}\right|_{2}^{1 / 2}\right) .
$$

Plugging this last estimate in (2.16) and (2.17) and summing lead to :

$$
\begin{gathered}
\frac{d}{d t}\left(\left|v_{\eta}\right|_{2}^{2}+\left|\partial_{x} v_{\eta}\right|_{2}^{2}\right)+\frac{\varepsilon}{2} f_{\min }^{\eta}\left(\left|\partial_{x} v_{\eta}\right|_{2}^{2}+\left|\partial_{x x}^{2} v_{\eta}\right|_{2}^{2}\right) \\
\leq C_{\varepsilon, \eta}\left(1+\left|v_{\eta}\right|_{2}^{6}+\left|\partial_{x} v_{\eta}\right|_{2}^{6}\right)
\end{gathered}
$$

which yields a local a priori bound in $L^{\infty}\left(0, T_{\eta} ; H^{1}\right) \cap L^{2}\left(0, T_{\eta} ; H^{2}\right)$ depending only on $\left|u_{0}\right|_{H^{1}}, \varepsilon, f_{\min }^{\eta}, f_{\max }^{\eta}, f_{\max }^{\eta \prime}$. These a priori bounds on $v_{\eta}$ give, thanks to (2.18) an estimate on $\dot{\xi}_{\eta}$ in $L^{4}\left(0, T_{\eta}\right)$. We therefore have proved:

Proposition 2.1 For any $u_{0}$ satisfying (2.5), there exist $T_{\eta}$ and a unique solution $u_{\eta}$ belonging to $\mathcal{C}\left(\left[0, T_{\eta}\right] ; H^{1}(] 0, L[)\right) \cap L^{2}\left(0, T_{\eta} ; H^{2}(] 0, L[)\right)$ and $\xi_{\eta}$ in $W^{1,4}\left(0, T_{\eta}\right)$ solution to (2.6)(2.9). Moreover, $T_{\eta}$ depends only on $\left|u_{0}\right|_{H^{1}}, \varepsilon, f_{\min }^{\eta}, f_{\max }^{\eta}, f_{\max }^{\eta \prime}$.

- Maximum principle : we will prove

Proposition 2.2 The solution $\left(u_{\eta}, \xi_{\eta}\right)$ given by proposition 2.1 satisfies :

$$
\begin{gathered}
\left.\alpha \leq u_{\eta}(t, x) \leq \max _{x \in] 0, L[} u_{0}(x), \quad \forall x \in\right] 0, L\left[, \quad \forall t \in\left[0, T_{\eta}\right]\right. \\
0 \leq \partial_{x} u_{\eta}(t, 0), \quad \dot{\xi}_{\eta}(t) \leq \alpha, \quad \forall t \in\left[0, T_{\eta}\right] .
\end{gathered}
$$


Proof : we work directly on (2.6)-(2.9).

We begin with showing that $\alpha \leq u_{\eta}$. The function $w_{\eta} \equiv u_{\eta}-\alpha$ satisfies

$$
\partial_{t} w_{\eta}-\dot{\xi}_{\eta}(t) \partial_{x} w_{\eta}+\partial_{x}\left(w_{\eta}+\alpha\right)^{2}-\varepsilon \partial_{x}\left(f_{\eta}\left(u_{\eta}\right) \partial_{x} w_{\eta}\right)=0 .
$$

Multiplying (2.20) by $-w_{\eta}^{-}$(where $g^{-}$denotes the negative part of $g, g=g^{+}-g^{-}$) leads to

$$
\frac{1}{2} \frac{d}{d t}\left|w_{\eta}^{-}\right|_{2}^{2}-\frac{\dot{\xi}_{\eta}(t)-2 \alpha}{2}\left(w_{\eta}^{-}(t, L)\right)^{2}-\frac{2}{3}\left(w_{\eta}^{-}(t, L)\right)^{3}+\varepsilon f_{\text {min }}^{\eta}\left|\partial_{x} w_{\eta}^{-}\right|_{2}^{2} \leq 0
$$

that is

$$
\frac{1}{2} \frac{d}{d t}\left|w_{\eta}^{-}\right|_{2}^{2}+\varepsilon f_{m i n}^{\eta}\left|\partial_{x} w_{\eta}^{-}\right|_{2}^{2} \leq C\left(\left|\dot{\xi}_{\eta}(t)\right|^{2}+\left|w_{\eta}^{-}\right|_{\infty}\right)\left|w_{\eta}^{-}\right|_{2}^{2} .
$$

Since $t \rightarrow\left|\dot{\xi}_{\eta}(t)\right|$ and $t \rightarrow\left|w_{\eta}^{-}(t)\right|_{\infty}$ are at least integrable in time and since $w_{\eta}^{-}(0, x) \equiv 0$, Gronwall's lemma implies $w_{\eta}^{-} \equiv 0$. That is $\alpha \leq u_{\eta}(t, x)$.

On the other hand, since $u_{\eta}(t, 0)=\alpha$ and $\alpha \leq u_{\eta}(t, x)$ one has $0 \leq \partial_{x} u_{\eta}(t, 0)$.

In the same way, denoted by $M_{0}=\max _{x \in] 0, L[} u_{0}(x) \geq 1$ and consider now $w_{\eta}=u_{\eta}-M_{0}$, we find that $w_{\eta}^{+}$satisfies the following equality :

$$
\frac{1}{2} \frac{d}{d t}\left|w_{\eta}^{+}\right|_{2}^{2}-\frac{\dot{\xi}_{\eta}(t)+2 M_{0}}{2}\left(w_{\eta}^{+}(t, L)\right)^{2}-\frac{2}{3}\left(w_{\eta}^{+}(t, L)\right)^{3}+\varepsilon f_{m i n}^{\eta}\left|\partial_{x} w_{\eta}^{+}\right|_{2}^{2}=0 .
$$

The same argument as above implies $u_{\eta} \leq M_{0}$, which ends the proof of proposition (2.2).

On one hand, thanks to proposition 2.1, $T_{\eta}$ depends only on $\left|u_{0}\right|_{H^{1}}, \varepsilon, f_{\min }^{\eta}, f_{\text {max }}^{\eta}, f_{\text {max }}^{\eta \prime}$. Therefore the maximum principle given in proposition 2.2 implies that the quantities $f_{\min }^{\eta}, f_{\max }^{\eta}, f_{\max }^{\eta \prime}$ are controlled indenpendently of $\eta$ and $t$. Therefore there exists $T_{0}>0$ such that $\forall \eta>0$, $T_{\eta} \geq T_{0}$. On the other hand, as the constant in (2.19) depends only on $\varepsilon, f_{\min }^{\eta}, f_{\max }^{\eta}, f_{\max }^{\eta \prime}$ it is straightforward to perform the limit $\eta \rightarrow 0$ since $\partial_{t} u_{\eta}$ is bounded in $L^{\infty}\left(0, T_{0} ; L^{2}\right)$. Thereby, the limit $(u, \xi)$ is a solution to

$$
\begin{gathered}
\left.\partial_{t} u-\dot{\xi}(t) \partial_{x} u+\partial_{x}\left(u^{2}-\varepsilon \sqrt{u} \partial_{x} u\right)=0, \quad \forall t, 0 \leq t \leq T_{0}, \quad \forall x \in\right] 0, L[, \\
\dot{\xi}(t)=\alpha-\frac{\varepsilon}{\sqrt{\alpha}} \partial_{x} u(t, 0), \quad u(t, 0)=\alpha, \quad \partial_{x} u(t, L)=0, \quad \forall t \in\left[0, T_{0}\right], \\
\left.u(0, x)=u_{0}(x), \quad x \in\right] 0, L[, \\
\left.\alpha \leq u(t, x) \forall t, 0 \leq t \leq T_{0}, \quad \forall x \in\right] 0, L[, \\
u \in \mathcal{C}\left(\left[0, T_{0}\right] ; H^{1}\right) \cap L^{2}\left(0, T_{0} ; H^{2}\right), \\
\xi \in W^{1,4}\left(0, T_{0}\right) .
\end{gathered}
$$

Now it is clear that any solution of the previous system (2.22)-(2.27) satisfies the conclusion of the proposition 2.2 (that is the maximum principle). Now performing the analogous of estimate (2.19) for the difference of two solutions of (2.22)-(2.27) yields uniqueness.

We therefore have proved :

Proposition 2.3 Let $u_{0} \in H^{1}(] 0, L[)$ such that $\left.\forall x \in\right] 0, L\left[, \quad 0<\alpha \leq u_{0}(x)\right.$ and $u_{0}(0)=\alpha$. There exist a time $T_{0}>0$ and a unique solution

$$
u \in \mathcal{C}\left(\left[0, T_{0}\right] ; H^{1}\right) \cap L^{2}\left(0, T_{0} ; H^{2}\right), \quad \dot{\xi} \in W^{1,4}\left(0, T_{0}\right)
$$

to (2.22)-(2.27). Moreover

$$
\begin{gathered}
\left.\alpha \leq u(t, x) \leq \max _{x \in] 0, L[} u_{0}(x), \quad \forall t \in\left[0, T_{0}\right], \quad \forall x \in\right] 0, L[ \\
\partial_{x} u(t, 0) \geq 0, \quad \dot{\xi}(t) \leq \alpha, \quad \forall t \in\left[0, T_{0}\right] .
\end{gathered}
$$

The next step is to show that if $u_{0}$ is more regular, then $u$ and $\dot{\xi}$ are also more regular.

- Regularity : we will prove : 
Proposition 2.4 Let $u_{0}$ as in proposition 2.3. Suppose moreover that $u_{0}$ belongs to $H^{3}(] 0, L[)$ and satisfies the compatibility condition

$$
-\dot{\xi}(0) \partial_{x} u_{0}(0)+\partial_{x}\left(u_{0}^{2}-\varepsilon \sqrt{u_{0}} \partial_{x} u_{0}\right)(x=0)=0 .
$$

Then, the unique solution given by proposition 2.3 satisfies

$$
u \in \mathcal{C}\left(\left[0, T_{0}\right] ; H^{3}\right) \cap L^{2}\left(0, T_{0} ; H^{4}\right), \quad \dot{\xi} \in W^{2,4}\left(0, T_{0}\right),
$$

and $|u|_{\mathcal{C}\left(\left[0, T_{0}\right] ; H^{3}\right) \cap L^{2}\left(0, T_{0} ; H^{4}\right)}$ depends only on $|u-\varphi|_{\mathcal{C}\left(\left[0, T_{0}\right] ; H^{1}\right) \cap L^{2}\left(0, T_{0} ; H^{2}\right)}$ and

$$
\varepsilon \sqrt{\alpha} \partial_{x x}^{2} u(t, 0)=\varepsilon \frac{1}{2 \sqrt{\alpha}}\left(\partial_{x} u(t, 0)\right)^{2}+\alpha \partial_{x} u(t, 0) \geq 0, \forall t \in\left[0, T_{0}\right] .
$$

Remark 2.1 The proof of this proposition shows that $T_{0}$ is the same as the one given by proposition 2.3, so that in order to obtain $T_{0}=+\infty$, it will be enough to have a "global" a priori estimate for $u$.

Proof: As usual, it is enough to prove some a priori estimates. Let $w=\partial_{t} u$. We will obtain some estimates on $\partial_{t} u$ which is equivalent to $\partial_{x x}^{2} u$ in terms of regularity. We differentiate (2.22) with respect to $t$ and multiply by $w$. Using the fact that $w(t, 0)=0$ and $\partial_{x} w(t, L)=0$, one gets

$$
\begin{gathered}
\frac{1}{2} \frac{d}{d t}|w|_{2}^{2}-\frac{1}{2} \dot{\xi}(t) w(t, L)^{2}-\ddot{\xi}(t) \int_{] 0, L[} \partial_{x} u w+2 \int_{] 0, L[} w^{2} \partial_{x} u \\
\quad+\int_{] 0, L[} u \partial_{x}\left(w^{2}\right)+\varepsilon \sqrt{\alpha}\left|\partial_{x} w\right|_{2}^{2} \leq \varepsilon\left|\int_{] 0, L[} \frac{\partial_{x} u}{2 \sqrt{u}} w \partial_{x} w\right|,
\end{gathered}
$$

which gives, using the $L^{\infty}$ bound of $u$

$$
\frac{d}{d t}|w|_{2}^{2}+\varepsilon \sqrt{\alpha}\left|\partial_{x} w\right|_{2}^{2} \leq|\dot{\xi}(t)|^{2}+|\ddot{\xi}(t)|^{2}+C|w|_{2}^{2}\left(1+\left|\partial_{x} u\right|_{\infty}^{2}+\left|\partial_{x} u\right|_{2}^{2}\right),
$$

where the constant $C$, depends only on $\varepsilon, \alpha$, and $M_{0}$. We now perform an $H^{1}$ estimate. Differentiating (2.22) with respect to $t$ and multiplying by $\partial_{x x}^{2} w$ lead to

$$
\begin{gathered}
\frac{1}{2} \frac{d}{d t}\left|\partial_{x} w\right|_{2}^{2}+\frac{1}{2} \dot{\xi}(t)\left|\partial_{x} w(t, 0)\right|_{2}^{2}+\ddot{\xi}(t) \int_{] 0, L[} \partial_{x} u \partial_{x x}^{2} w+2 \int_{] 0, L[} w \partial_{x} u \partial_{x x}^{2} w \\
+\varepsilon \int_{] 0, L[} \sqrt{u}\left(\partial_{x x}^{2} w\right)^{2}+\varepsilon \int_{] 0, L[} \frac{1}{\sqrt{u}} \partial_{x} u \partial_{x} w \partial_{x x}^{2} w \\
-\varepsilon \int_{] 0, L[} \frac{w}{4 u^{3 / 2}}\left(\partial_{x} u\right)^{2} \partial_{x x}^{2} w+\varepsilon \int_{] 0, L[} \frac{w}{2 \sqrt{u}} \partial_{x x}^{2} u \partial_{x x}^{2} w=0
\end{gathered}
$$

Using Young's inequality and (2.15) yields

$$
\begin{gathered}
\frac{d}{d t}\left|\partial_{x} w\right|_{2}^{2}+\varepsilon \sqrt{\alpha}\left|\partial_{x x}^{2} w\right|_{2}^{2} \leq \ddot{\xi}^{2}(t)\left|\partial_{x} u\right|_{2}^{2} \\
+C\left(|w|_{2}^{2}+\left|\partial_{x} w\right|_{2}^{2}\right)\left(\dot{\xi}^{2}(t)+\left|\partial_{x} u\right|_{\infty}^{2}+\left|\partial_{x} u\right|_{\infty}^{4}+\left|\partial_{x x}^{2} u\right|_{2}^{2}\right),
\end{gathered}
$$

where the constant $C$ depends only on $\varepsilon$.

Now, we write $\ddot{\xi}(t)=-\frac{\varepsilon}{\sqrt{\alpha}} \partial_{x} w(t, 0)$, so that

$$
|\ddot{\xi}(t)| \leq C\left|\partial_{x} w\right|_{2}^{1 / 2}\left|\partial_{x x}^{2} w\right|_{2}^{1 / 2},
$$


therefore (2.29) and (2.30) give ;

$$
\begin{aligned}
& \frac{d}{d t}\left(|w|_{2}^{2}+\left|\partial_{x} w\right|_{2}^{2}\right)+\frac{\varepsilon \sqrt{\alpha}}{2}\left(\left|\partial_{x} w\right|_{2}^{2}+\left|\partial_{x x}^{2} w\right|_{2}^{2}\right) \\
& \leq C\left(1+\left|\partial_{x} u\right|_{\infty}^{4}+\left|\partial_{x x}^{2} u\right|_{2}^{2}\right)\left(|w|_{2}^{2}+\left|\partial_{x} w\right|_{2}^{2}\right)
\end{aligned}
$$

Since $t \rightarrow\left|\partial_{x} u\right|_{\infty}^{4}+\left|\partial_{x x}^{2} u\right|_{2}^{2}$ is time-integrable, we get, using Gronwall lemma,

$$
|w|_{L^{\infty}\left(0, T_{0} ; H^{1}\right) \cap L^{2}\left(0, T_{0} ; H^{2}\right)} \leq C\left(T_{0}\right)\left(|w(0)|_{2}+\left|\partial_{x} w(0)\right|_{2}\right)
$$

where $C\left(T_{0}\right)$ depends only on $|u|_{L^{\infty}\left(\left(0, T_{0}\right) \times(0, L)\right)}$ and $\left|\partial_{x} u\right|_{L^{\infty}\left(0, T_{0} ; L^{2}\right) \cap L^{2}\left(0, T_{0} ; H^{1}\right)}$, thereby proving the first part of the proposition.

Writting equation (2.22) at $x=0$ leads to

$$
-\dot{\xi}(t) \partial_{x} u(t, 0)+2 \alpha \partial_{x} u(t, 0)-\varepsilon \sqrt{\alpha} \partial_{x x}^{2} u(t, 0)-\frac{\varepsilon}{2 \sqrt{\alpha}}\left(\partial_{x} u(t, 0)\right)^{2}=0 .
$$

Recalling that $\dot{\xi}(t)=\alpha-\frac{\varepsilon}{\sqrt{\alpha}} \partial_{x} u(t, 0)$, one gets

$$
\varepsilon \sqrt{\alpha} \partial_{x x}^{2} u(t, 0)=\frac{\varepsilon}{2 \sqrt{\alpha}}\left(\partial_{x} u(t, 0)\right)^{2}+\alpha \partial_{x} u(t, 0),
$$

which ends the proof of the proposition 2.4.

\subsection{Oleinik-like condition}

The aim of this section is to find an upper bound (independent of $\varepsilon$ ) for $\partial_{x} u$ and to show that if $\partial_{x} u_{0} \geq 0$ then $\partial_{x} u(t, x)$ remains non-negative for all time $t$.

Proposition 2.5 The solution $(u, \xi)$ given by proposition 2.4 satisfies

$$
\left.\partial_{x} u(t, x) \leq \frac{1}{\frac{1}{\max \partial_{x} u_{0}(x)}+2 t}, \quad \forall x \in\right] 0, L\left[, \quad \forall t \in\left[0, T_{0}\right],\right.
$$

and

$$
\alpha-\frac{\varepsilon}{\sqrt{\alpha}} \frac{1}{\frac{1}{\max \partial_{x} u_{0}(x)}+2 t} \leq \dot{\xi}(t) \leq \alpha, \quad \forall t \in\left[0, T_{0}\right]
$$

Proof : We denote by $M_{1}(t)=\frac{1}{\frac{1}{\max \partial_{x} u_{0}(x)}+2 t}$ and $w(t, x)=\partial_{x} u(t, x)-M_{1}(t)$. Since $u_{0}(0)=\alpha$ and $u_{0}(x) \geq \alpha$ for all $x \in[0, L]$, max $\partial_{x} u_{0}(x) \geq 0$ and therefore function $M_{1}$ is well-defined for $t>0$. Moreover $M_{1}^{\prime}(t)=-2 M_{1}(t)^{2}$. The equation satisfied by $w$ reads

$$
\begin{gathered}
\partial_{t} w+M_{1}^{\prime}-\dot{\xi} \partial_{x} w+2\left(w+M_{1}\right)^{2}+2 u \partial_{x} w \\
-\varepsilon \partial_{x}\left(\frac{1}{2 \sqrt{u}}\left(w+M_{1}\right)^{2}+\sqrt{u} \partial_{x} w\right)=0 .
\end{gathered}
$$


Taking the $L^{2}$ inner product of the equation above by $w^{+}$gives $\left(\right.$as $\left.w^{+}(L, t)=0\right)$

$$
\begin{gathered}
\frac{1}{2} \frac{d}{d t}\left|w^{+}\right|_{2}^{2}+M_{1}^{\prime}(t) \int_{] 0, L[} w^{+}+\frac{1}{2} \dot{\xi}(t)\left(w^{+}(t, 0)\right)^{2} \\
+2 \int_{] 0, L[} w^{+}\left(w+M_{1}(t)\right)^{2}+\int_{] 0, L[} u \partial_{x}\left(w^{+}\right)^{2} \\
+\varepsilon \int_{] 0, L[} \frac{1}{4 u^{3 / 2}} \partial_{x} u\left(w+M_{1}(t)\right)^{2} w^{+} \\
-\varepsilon \int_{] 0, L[} \frac{1}{2 \sqrt{u}}\left(\partial_{x}\left(w^{2}\right)+2 M_{1}(t) \partial_{x} w\right) w^{+} \\
+\varepsilon \int_{] 0, L[} \sqrt{u}\left(\partial_{x} w^{+}\right)^{2}-\varepsilon\left[\sqrt{u} \partial_{x} w w^{+}\right]_{0}^{L}=0
\end{gathered}
$$

We bound $\left(w^{+}(t, 0)\right)^{2}$ by $\sqrt{2}\left|w^{+}\right|_{2}\left|\partial_{x} w\right|_{2}$. On one hand the term $\int_{] 0, L[} u \partial_{x}\left(w^{+}\right)^{2}$ is controlled by $|u|_{\infty}\left|w^{+}\right|_{2}\left|\partial_{x} w^{+}\right|_{2}$. On the other hand,

$$
\begin{aligned}
& \int_{] 0, L[} \frac{1}{4 u^{3 / 2}} \partial_{x} u\left(w+M_{1}(t)\right)^{2} w^{+}=\int_{] 0, L[} \frac{1}{4 u^{3 / 2}}\left(w+M_{1}(t)\right)\left(w+M_{1}(t)\right)^{2} w^{+} \\
& =M_{1}(t) \int_{] 0, L[} \frac{1}{4 u^{3 / 2}}\left(w+M_{1}(t)\right)^{2} w^{+}+\int_{] 0, L[} \frac{1}{4 u^{3 / 2}}\left(w^{+}\right)^{2}\left(w+M_{1}(t)\right)^{2} \geq 0
\end{aligned}
$$

The term $\varepsilon \int_{] 0, L[} \frac{1}{2 \sqrt{u}}\left(\partial_{x}\left(w^{2}\right)+2 M_{1}(t) \partial_{x} w\right) w^{+}$is controled by

$C\left(1+|w|_{\infty}\right)\left|w^{+}\right|_{2}\left|\partial_{x} w^{+}\right|_{2}$. Finally, one has $\sqrt{u} \partial_{x} w w^{+}(t, 0) \geq 0$ thanks to (2.28).

We therefore obtain

$$
\begin{gathered}
\frac{1}{2} \frac{d}{d t}\left|w^{+}\right|_{2}^{2}+\sqrt{\alpha} \frac{\varepsilon}{2}\left|\partial_{x} w^{+}\right|_{2}^{2}+\int_{] 0, L[}\left(M_{1}^{\prime}(t)+2\left(w+M_{1}(t)\right)^{2}\right) w^{+} \\
\leq C\left(1+|\dot{\xi}(t)|+|w|_{\infty}^{2}\right)\left|w^{+}\right|_{2}^{2} .
\end{gathered}
$$

According to the expression of $M_{1}^{\prime}(t)$, we find

$$
\begin{aligned}
\int_{] 0, L[}\left(M_{1}^{\prime}(t)+2\left(w+M_{1}(t)\right)^{2}\right) w^{+} & =\int_{] 0, L[}\left(M_{1}^{\prime}(t)+2\left(w^{2}+2 w M_{1}(t)+M_{1}^{2}(t)\right)\right) w^{+} \\
& =2 \int_{] 0, L[}\left(w^{2}+2 w M_{1}(t)\right) w^{+} \geq 0 .
\end{aligned}
$$

The inequality above leads to

$$
\frac{1}{2} \frac{d}{d t}\left|w^{+}\right|_{2}^{2}+\sqrt{\alpha} \frac{\varepsilon}{2}\left|\partial_{x} w^{+}\right|_{2}^{2} \leq C\left(1+|\dot{\xi}(t)|+|w|_{\infty}^{2}\right)\left|w^{+}\right|_{2}^{2} .
$$

Gronwall's lemma implies then $w^{+} \equiv 0$. The inequality on $\dot{\xi}$ follows.

Moreover, one has:

Proposition 2.6 Suppose $\left.\partial_{x} u_{0}(x) \geq 0, \quad \forall x \in\right] 0, L[$. Then the solution $(u, \xi)$ given by proposition 2.4 satisfies

$$
\left.\partial_{x} u(t, x) \geq 0, \quad \forall x \in\right] 0, L\left[, \quad \forall t \in\left[0, T_{0}\right]\right.
$$


Proof : Let us denote $w=\partial_{x} u$. The equation satisfied by $w$ reads:

$$
\partial_{t} w-\dot{\xi} \partial_{x} w+2 w^{2}+2 u \partial_{x} w-\varepsilon \partial_{x}\left(\frac{1}{2 \sqrt{u}} w^{2}+\sqrt{u} \partial_{x} w\right)=0 .
$$

One multiplies this equation by $-w^{-}$to obtain

$\frac{1}{2} \frac{d}{d t}\left|w^{-}\right|_{2}^{2}-2 \int_{] 0, L[}\left(w^{-}\right)^{3}+\int_{0, L[} u \partial_{x}\left(w^{-}\right)^{2}-\varepsilon \int_{0, L[} \frac{1}{2 \sqrt{u}}\left(w^{-}\right)^{2} \partial_{x} w^{-}+\varepsilon \int_{0, L[} \sqrt{u}\left(\partial_{x} w^{-}\right)^{2}=0$, since $w^{-}(t, 0)=w^{-}(t, L)=0$. Finally we get

which leads to the result.

$$
\frac{d}{d t}\left|w^{-}\right|_{2}^{2}+\varepsilon \sqrt{\alpha}\left|\partial_{x} w^{-}\right|_{2}^{2} \leq C\left(1+|w|_{\infty}^{2}\right)\left|w^{-}\right|_{2}^{2}
$$

\subsection{End of proof of theorem 1}

Now using the estimate on $\dot{\xi}$ and on $|u|_{\infty}$, the inequality (2.14) written for $\eta=0$ reads:

$$
\frac{1}{2} \frac{d}{d t}|v|_{2}^{2}+\varepsilon \sqrt{\alpha}\left|\partial_{x} v\right|_{2}^{2} \leq C\left(1+|v|_{2}^{2}\right)
$$

where $C$ depends only on $\alpha,\left|u_{0}\right|_{W^{1, \infty}}$ and not on $\varepsilon$ and $L$.

Now we perform an $H^{1}$-estimate on $u$.

One multiplies (2.1) by $-\partial_{x x}^{2} u$ to get

$$
\begin{gathered}
\frac{1}{2} \frac{d}{d t}\left|\partial_{x} u\right|_{2}^{2}+\frac{\dot{\xi}(t)}{2}\left[\left(\partial_{x} u\right)^{2}\right]_{0}^{L}-2 \int_{] 0, L[} u \partial_{x} u \partial_{x x}^{2} u \\
+\varepsilon \sqrt{\alpha}\left|\partial_{x x}^{2} u\right|_{2}^{2}+\frac{\varepsilon}{2} \int_{0, L[} \frac{1}{\sqrt{u}}\left(\partial_{x} u\right)^{2} \partial_{x x}^{2} u \leq 0
\end{gathered}
$$

We remark that

$$
\begin{gathered}
\int_{] 0, L[} \frac{1}{\sqrt{u}}\left(\partial_{x} u\right)^{2} \partial_{x x}^{2} u=\frac{1}{3} \int_{] 0, L[} \frac{1}{\sqrt{u}} \partial_{x}\left(\left(\partial_{x} u\right)^{3}\right) \\
=\frac{1}{6} \int_{] 0, L[} \frac{1}{u^{3 / 2}}\left(\partial_{x} u\right)^{4}+\left[\frac{1}{3 \sqrt{u}}\left(\partial_{x} u\right)^{3}\right]_{0}^{L} \geq-\frac{1}{3 \sqrt{\alpha}}\left(\partial_{x} u(t, 0)\right)^{3} .
\end{gathered}
$$

We therefore obtain

$$
\frac{d}{d t}\left|\partial_{x} u\right|_{2}^{2}+\varepsilon \sqrt{\alpha}\left|\partial_{x x}^{2} u\right|_{2}^{2} \leq C_{\varepsilon}\left(1+\left|\partial_{x} u\right|_{2}^{2}+\left(\partial_{x} u(t, 0)\right)^{3}\right)
$$

Now, thanks to the Oleinik property given in proposition 2.5 , one has $\left(\partial_{x} u(t, 0)\right)^{3} \leq\left(\max _{x \in] 0, L[} \partial_{x} u_{0}\right)^{3}$.

Inequalities (2.32) and (2.34) imply that there exists a continuous function $g$ depending on $\varepsilon$ but not on $L$ such that

$$
\begin{gathered}
|u(t)-\varphi|_{2}^{2}+\left|\partial_{x} u(t)\right|_{2}^{2}+\int_{0}^{t}\left|\partial_{x} u(s)\right|_{2}^{2}+\left|\partial_{x x}^{2} u(s)\right|_{2}^{2} d s \\
\leq g(t)\left(1+\left|u_{0}-\varphi\right|_{2}^{2}+\left|\partial_{x} u_{0}\right|_{2}^{2}\right), \quad \forall t \in\left[0, T_{0}\right] .
\end{gathered}
$$

Using proposition 2.4, one gets a bound for $u$ in $\mathcal{C}\left(\left[0, T_{0}\right] ; H^{3}\right) \cap L^{2}\left(0, T_{0} ; H^{4}\right)$. Therefore the solution is global. Moreover, all bounds are independent of $L$, so that we can perform the limit $L \rightarrow \infty$.

If $u_{0}$ lies only in $H^{1} \cap W^{1, \infty}$, one takes a sequence $\left(u_{0}^{n}-\varphi\right)_{n} \in H^{3} \cap W^{1, \infty}$ satisfying the compatibility condition of proposition 2.4 such that $u_{0}^{n}-\varphi \rightarrow u_{0}-\varphi$ in $H^{1}$ strongly and in $W^{1, \infty}$ weakly $\star$. Thanks to the estimates $(2.32),(2.34)$ one obtains global solutions. 


\section{Convergence towards Burger equation.}

The aim of this section is to prove the following result.

\section{Theorem 3.1}

- Let $u_{0} \in B V(] 0, L[)$ satisfy, $\left.\alpha \leq u_{0}(x), \quad \forall x \in\right] 0, L\left[\right.$. Let $u_{0}^{\varepsilon} \in W^{1, \infty}(] 0, L[)$ satisfy (2.5) such that $u_{0}^{\varepsilon} \rightarrow u_{0}$ in $B V(] 0, L[)$ and $\left|\partial_{x} u_{0}^{\varepsilon}\right|_{\infty}=o\left(\frac{1}{\varepsilon}\right)$. The corresponding solution $\left(u^{\varepsilon}, \xi^{\varepsilon}\right)$ of (2.1)-(2.4) given by theorem 2.1 satisfies

$$
\dot{\xi}^{\varepsilon} \rightarrow_{\varepsilon \rightarrow 0} \alpha \text { in } L^{\infty}\left(\mathbb{R}_{+}\right), u^{\varepsilon} \rightarrow_{\varepsilon \rightarrow 0} u \text { in } L_{l o c}^{2}\left(\mathbb{R}^{+} ; L^{2}(] 0, L[)\right),
$$

where $u \in L^{\infty}\left(\mathbb{R}^{+} \times\right] 0, L[) \cap L^{\infty}\left(\mathbb{R}_{+} ; B V(] 0, L[)\right)$ is the unique entropy solution to

$$
\begin{gathered}
\left.\partial_{t} u-\alpha \partial_{x} u+\partial_{x}\left(u^{2}\right)=0, \quad \forall t \in \mathbb{R}^{+}, \quad \forall x \in\right] 0, L[, \\
u(t, 0)=\alpha, \quad \forall t \in \mathbb{R}^{+}, \\
\left.u(0, x)=u_{0}(x), \quad \forall x \in\right] 0, L[.
\end{gathered}
$$

- Suppose moreover that $u_{0} \in W^{1, \infty}(] 0, L[)$ and $\partial_{x} u_{0} \geq 0$ a.e.. Then the solution given by theorem 2.1 satisfies

$$
u^{\varepsilon} \rightarrow_{\varepsilon \rightarrow 0} u \text { in } \mathcal{C}\left(\mathbb{R}^{+} \times\right] 0, L[) \text { and in } L^{\infty}\left(\mathbb{R}^{+} ; W^{1, \infty}(] 0, L[)\right) \text { weak } \star
$$

\subsection{Monotone case}

In this section, we deal with non-decreasing initial data. We suppose moreover that $u_{0}$ belongs to $W^{1, \infty}(] 0, L[)$ and verifies $u_{0}(0)=\alpha$. In this case we prove

Proposition 3.1 Let $u_{0} \in W^{1, \infty}(] 0, L[)$ satisfy (2.5). Suppose moreover that $\partial_{x} u_{0}$ is nonnegative a.e. Then the solution $\left(u^{\varepsilon}, \xi^{\varepsilon}\right)$ given by theorem 2.1 satisfies

$$
\begin{gathered}
u^{\varepsilon} \rightarrow_{\varepsilon \rightarrow 0} u \text { in } \mathcal{C}\left(\mathbb{R}^{+} \times\right] 0, L[) \text { and in } L^{\infty}\left(\mathbb{R}^{+} ; W^{1, \infty}(] 0, L[)\right) \text { weak } \star \\
\dot{\xi}^{\varepsilon} \rightarrow_{\varepsilon \rightarrow 0} \alpha \text { in } L^{\infty}\left(\mathbb{R}^{+}\right),
\end{gathered}
$$

where $u$ is the unique entropy solution to

$$
\begin{gathered}
\left.\partial_{t} u-\alpha \partial_{x} u+\partial_{x}\left(u^{2}\right)=0, \quad \forall t \in \mathbb{R}^{+}, \quad \forall x \in\right] 0, L[, \\
u(t, 0)=\alpha, \quad \forall t \in \mathbb{R}^{+}, \\
\left.u(0, x)=u_{0}(x), \quad \forall x \in\right] 0, L[.
\end{gathered}
$$

Proof: Thanks to theorem 2.1, since $\partial_{x} u_{0} \geq 0$, a.e. one has

$$
0 \leq \partial_{x} u^{\varepsilon}(t, x) \leq \frac{1}{\frac{1}{\max \partial_{x} u_{0}}+2 t}
$$

which yields a bound for $u^{\varepsilon}$ in $L^{\infty}\left(\mathbb{R}^{+} ; W^{1, \infty}(] 0, L[)\right)$. Moreover, it follows readly from theorem 2.1 that $\dot{\xi}^{\varepsilon} \rightarrow_{\varepsilon \rightarrow 0} \alpha$ in $L^{\infty}\left(\mathbb{R}^{+}\right)$.

Coming back to $(2.32)$ yields a bound for $u^{\varepsilon}-1$ in $L_{l o c}^{\infty}\left(\mathbb{R}^{+} ; L^{2}(] 0, L[)\right)$. Now rewritting $(2.33)$ as

$$
\begin{aligned}
& \frac{1}{2} \frac{d}{d t}\left|\partial_{x} u^{\varepsilon}\right|_{2}^{2}+\int_{] 0, L[}\left(\partial_{x} u^{\varepsilon}\right)^{3}+\frac{\dot{\xi}(t)}{2}\left[\left(\partial_{x} u^{\varepsilon}\right)^{2}\right]_{0}^{L}-\left[u\left(\partial_{x} u^{\varepsilon}\right)^{2}\right]_{0}^{L} \\
& +\frac{\varepsilon}{12} \int_{] 0, L[} \frac{1}{\left(u^{\varepsilon}\right)^{3 / 2}}\left(\partial_{x} u^{\varepsilon}\right)^{4}+\frac{\varepsilon}{6}\left[\frac{\left(\partial_{x} u^{\varepsilon}\right)^{3}}{\sqrt{u^{\varepsilon}}}\right]_{0}^{L}+\varepsilon \sqrt{\alpha}\left|\partial_{x x}^{2} u^{\varepsilon}\right|_{2}^{2} \leq 0 .
\end{aligned}
$$


Using the $L^{\infty}$ bound on $\partial_{x} u^{\varepsilon}$ and $\partial_{x} u^{\varepsilon} \geq 0$ gives a bound for $\left|\partial_{x} u^{\varepsilon}\right|_{L_{l o c}^{\infty}\left(\mathbb{R}^{+} ; L^{2}\right)}$ and for $\sqrt{\varepsilon}\left|\partial_{x x}^{2} u^{\varepsilon}\right|_{L_{\text {loc }}^{2}\left(\mathbb{R}_{;}^{+} ; L^{2}\right)}$ independant of $\varepsilon$ and $L$. This implies that $\partial_{t} u^{\varepsilon}$ is bounded in $L_{\text {loc }}^{2}\left(\mathbb{R}^{+} ; L^{2}\right)$. Aubin's lemma gives that the sequence $\left(u^{\varepsilon}\right)_{\varepsilon}$ is compact in $\mathcal{C}^{0}([0, T] \times] 0, L[)$ and in $L^{\infty}\left(\mathbb{R}^{+} ; W^{1, \infty}(] 0, L[)\right)$ weak $\star$, which is enough to perform the limit. Of course, the limit $u$ satisfies Oleinik entropy condition and is therefore unique [5], so that all the sequence $u^{\varepsilon}$ converges. Note that the limit $u$ lies in fact in $W^{1, \infty}\left(\mathbb{R}^{+} \times\right] 0, L[)$.

Remark 3.1 The result of proposition 3.1 hold for $L=+\infty$, with strong convergence for $u^{\varepsilon}$ in $\mathcal{C}([0, T] \times[0, X])$, for all $T>0, X>0$.

\subsection{General case}

In this section we do not suppose that $u_{0}$ is non-decreasing. This implies that one can not obtain some $W^{1, \infty}$ bounds for $u$, since at the limit, some shocks may occur. We will prove

Proposition 3.2 Let $u_{0} \in B V(] 0, L[)$ satisfy $\left.\alpha \leq u_{0}(x), \forall x \in\right] 0, L\left[\right.$. Let $u_{0}^{\varepsilon} \in W^{1, \infty}(] 0, L[)$ satisfy (2.5) such that $u_{0}^{\varepsilon} \rightarrow_{\varepsilon \rightarrow 0} u_{0}$ in $B V(] 0, L[)$ and $\left|\partial_{x} u_{0}^{\varepsilon}\right|_{\infty}=o\left(\frac{1}{\varepsilon}\right)$. Then the solution $\left(u^{\varepsilon}, \xi^{\varepsilon}\right)$ of (2.1)-(2.4) given by theorem 2.1 satisfies

$$
\begin{gathered}
\dot{\xi}^{\varepsilon} \rightarrow_{\varepsilon \rightarrow 0} \alpha \text { in } L^{\infty}\left(\mathbb{R}^{+}\right), \\
u^{\varepsilon} \rightarrow_{\varepsilon \rightarrow 0} u \text { in } L_{l o c}^{2}\left(\mathbb{R}^{+} ; L^{2}(] 0, L[)\right)
\end{gathered}
$$

where $u$ is the unique entropy solution to

$$
\begin{gathered}
\left.\partial_{t} u-\alpha \partial_{x} u+\partial_{x}\left(u^{2}\right)=0, \quad \forall t \in \mathbb{R}^{+}, \quad \forall x \in\right] 0, L[ \\
u(t, 0)=\alpha, \quad \forall t \in \mathbb{R}^{+} \\
\left.u(0, x)=u_{0}(x), \quad \forall x \in\right] 0, L[.
\end{gathered}
$$

Proof : Let $u_{0}^{\varepsilon} \in W^{1, \infty}(] 0, L[)$ such that $u_{0}^{\varepsilon} \rightarrow_{\varepsilon \rightarrow 0} u_{0}$ in $B V(] 0, L[)$, and suppose moreover that

$$
\left|\partial_{x} u_{0}^{\varepsilon}\right|_{\infty}=o\left(\frac{1}{\varepsilon}\right) .
$$

The equation satisfied by $\partial_{x} u^{\varepsilon}$ reads

$$
\begin{gathered}
\partial_{t} \partial_{x} u^{\varepsilon}-\dot{\xi}^{\varepsilon} \partial_{x x}^{2} u^{\varepsilon}+2 \partial_{x}\left(u^{\varepsilon} \partial_{x} u^{\varepsilon}\right) \\
-\varepsilon \partial_{x}\left(\frac{\left(\partial_{x} u^{\varepsilon}\right)^{2}}{2 \sqrt{u^{\varepsilon}}}\right)-\varepsilon \partial_{x}\left(\sqrt{u^{\varepsilon}} \partial_{x x}^{2} u^{\varepsilon}\right)=0 .
\end{gathered}
$$

Following [1], we introduce the regularization of sign function given by:

$$
\operatorname{sgn}_{\eta}(x)=\left\{\begin{array}{l}
1 \text { if } x \geq \eta \\
\frac{x}{\eta} \text { if }-\eta \leq x \leq \eta \\
-1 \quad \text { if } x \leq-\eta
\end{array}\right.
$$


and $F_{\eta}(x)=\int_{0}^{x} \operatorname{sgn}_{\eta}(y) d y$.

Multiplying (3.2) by $s g n_{\eta}\left(\partial_{x} u^{\varepsilon}\right)$ leads to, after integration

$$
\begin{aligned}
& \frac{d}{d t} \int_{] 0, L[} F_{\eta}\left(\partial_{x} u^{\varepsilon}\right)-\dot{\xi}(t) \int_{] 0, L[} \partial_{x}\left(F_{\eta}\left(\partial_{x} u^{\varepsilon}\right)\right) \\
& +2 \int_{] 0, L[}\left(\left(\partial_{x} u^{\varepsilon}\right)^{2} \operatorname{sgn}_{\eta}\left(\partial_{x} u^{\varepsilon}\right)+u^{\varepsilon} \partial_{x}\left(F_{\eta}\left(\partial_{x} u^{\varepsilon}\right)\right)\right) \\
& +\varepsilon \int_{] 0, L[} \sqrt{u^{\varepsilon}}\left(\partial_{x x}^{2} u^{\varepsilon}\right)^{2} s g n_{\eta}^{\prime}\left(\partial_{x} u^{\varepsilon}\right)-\varepsilon\left[\sqrt{u^{\varepsilon}} \partial_{x x}^{2} u^{\varepsilon} \operatorname{sgn}_{\eta}\left(\partial_{x} u^{\varepsilon}\right)\right]_{0}^{L} \\
& +\varepsilon \int_{] 0, L[} \frac{\left(\partial_{x} u^{\varepsilon}\right)^{2}}{2 \sqrt{u^{\varepsilon}}} \partial_{x x}^{2} u^{\varepsilon} \operatorname{sgn}_{\eta}^{\prime}\left(\partial_{x} u^{\varepsilon}\right)-\varepsilon\left[\frac{\left(\partial_{x} u^{\varepsilon}\right)^{2}}{2 \sqrt{u^{\varepsilon}}} \operatorname{sgn}_{\eta}\left(\partial_{x} u^{\varepsilon}\right)\right]_{0}^{L}=0
\end{aligned}
$$

On one hand $\partial_{x} u^{\varepsilon}(t, L)=0$, on the other hand $\partial_{x} u^{\varepsilon}(t, 0) \geq 0$.

One can control the term

$$
\varepsilon \int_{] 0, L[} \frac{\left(\partial_{x} u^{\varepsilon}\right)^{2}}{2 \sqrt{u^{\varepsilon}}} \partial_{x x}^{2} u^{\varepsilon} \operatorname{sgn}_{\eta}^{\prime}\left(\partial_{x} u^{\varepsilon}\right)
$$

by

$$
\varepsilon \int_{] 0, L[} \sqrt{u^{\varepsilon}}\left(\partial_{x x}^{2} u^{\varepsilon}\right)^{2} \operatorname{sgn}_{\eta}^{\prime}\left(\partial_{x} u^{\varepsilon}\right)+C \varepsilon \int_{] 0, L[} \frac{\left(\partial_{x} u^{\varepsilon}\right)^{4}}{u^{\varepsilon}} \operatorname{sgn}_{\eta}^{\prime}\left(\partial_{x} u^{\varepsilon}\right) .
$$

This last term is bounded by $C \varepsilon \eta^{3} L$. Therefore, (3.3) gives

$$
\begin{gathered}
\frac{d}{d t} \int_{] 0, L[} F_{\eta}\left(\partial_{x} u^{\varepsilon}\right)+\dot{\xi}(t) F_{\eta}\left(\partial_{x} u^{\varepsilon}(t, 0)\right) \\
+2 \int_{] 0, L[}\left(\left(\partial_{x} u^{\varepsilon}\right)^{2} s g n_{\eta}\left(\partial_{x} u^{\varepsilon}\right)-\partial_{x} u^{\varepsilon} F_{\eta}\left(\partial_{x} u^{\varepsilon}\right)\right) \\
-2 \alpha F_{\eta}\left(\partial_{x} u^{\varepsilon}(t, 0)\right)+\varepsilon \sqrt{\alpha} \partial_{x x}^{2} u^{\varepsilon}(t, 0) \operatorname{sgn}_{\eta}\left(\partial_{x} u^{\varepsilon}\left(\partial_{x} u^{\varepsilon}(t, 0)\right)\right. \\
+\varepsilon \frac{\left(\partial_{x} u^{\varepsilon}(t, 0)\right)^{2}}{2 \sqrt{\alpha}} \operatorname{sgn}_{\eta}\left(\partial_{x} u^{\varepsilon}(t, 0)\right) \leq C \varepsilon \eta^{3} L .
\end{gathered}
$$

Let $G_{\eta}=\frac{F_{\eta}}{s g n_{\eta}}$. The boundary term in (3.4) reads

$$
\begin{aligned}
& B_{\eta}=\left((\dot{\xi}-2 \alpha) G_{\eta}\left(\partial_{x} u^{\varepsilon}(t, 0)\right)+\varepsilon \sqrt{\alpha} \partial_{x x}^{2} u^{\varepsilon}(t, 0)+\varepsilon \frac{\left(\partial_{x} u^{\eta}(t, 0)\right)^{2}}{2 \sqrt{\alpha}}\right) \\
& \times \operatorname{sgn}_{\eta}\left(\partial_{x} u^{\varepsilon}(t, 0)\right)
\end{aligned}
$$

Writing equation (2.1) at $x=0$ gives

$$
(2 \alpha-\dot{\xi}(t)) \partial_{x} u^{\varepsilon}(t, 0)-\frac{\varepsilon}{2 \sqrt{\alpha}}\left(\partial_{x} u^{\varepsilon}(t, 0)\right)^{2}-\varepsilon \sqrt{\alpha} \partial_{x x}^{2} u^{\varepsilon}(t, 0)=0 .
$$

Letting $\eta \rightarrow 0$ in (3.5) leads to

$$
\begin{gathered}
B=\left((\dot{\xi}-2 \alpha) \partial_{x} u^{\varepsilon}(t, 0)+\varepsilon \sqrt{\alpha} \partial_{x x}^{2} u^{\varepsilon}(t, 0)+\frac{\varepsilon}{2 \sqrt{\alpha}}\left(\partial_{x} u^{\varepsilon}(t, 0)\right)^{2}\right) \\
\times \operatorname{sgn}\left(\partial_{x} u^{\varepsilon}(t, 0)\right) .
\end{gathered}
$$

with(3.6), we get $B=0$. 
On the other hand

$$
2 \int_{] 0, L[}\left(\left(\partial_{x} u^{\varepsilon}\right)^{2} \operatorname{sgn}_{\eta}\left(\partial_{x} u^{\varepsilon}\right)-\partial_{x} u^{\varepsilon} F_{\eta}\left(\partial_{x} u^{\varepsilon}\right)\right) \rightarrow_{\eta \rightarrow 0} 0
$$

so that (3.4) gives

$$
\frac{d}{d t} \int_{] 0, L[}\left|\partial_{x} u^{\varepsilon}\right| \leq 0
$$

Therefore, $u^{\varepsilon}$ is bounded in $L^{\infty}\left(\mathbb{R}^{+} ; B V(] 0, L[)\right)$. It is clear that $\partial_{t} u^{\varepsilon}$ is bounded in $L_{l o c}^{2}\left(\mathbb{R}^{+} ; H^{-1}(] 0, L[)\right)$. Thanks to condition (3.1), $\dot{\xi}^{\varepsilon} \rightarrow_{\varepsilon \rightarrow 0} \alpha$ in $L^{\infty}\left(\mathbb{R}^{+}\right)$, and that the sequence $\left(u^{\varepsilon}\right)_{\varepsilon}$ is compact in $L_{\text {loc }}^{2}\left(\mathbb{R}^{+} ; L^{2}(] 0, L[)\right.$ strong and in $\mathcal{C}\left(\mathbb{R}^{+} ; H^{-1}(] 0, L[)\right.$ which is enough to prove that the limit $u$ satisfies

$$
\begin{gathered}
\partial_{t} u-\alpha \partial_{x} u+\partial_{x}(u)^{2}=0 \\
u(0, x)=u_{0}(x) .
\end{gathered}
$$

Since the initial value satisfies $u_{0}(x) \geq \alpha$, the caracteristics at $x=0$ are allways incoming. Therefore as in [1], the boundary condition $u(t, 0)=\alpha$ is concerved through the limit process. On the other hand, extending function $u(t, x)$ for $x \leq 0$ by $u(t, x) \equiv \alpha$ leads to a global solution for Burgers equation on the whole line satisfying the Oleinik entropy condition. Which yields uniqueness. This ends the proof of proposition 3.2.

As before, the result is valid for $L=+\infty$ with straightforward modifications.

Note that if $\partial_{x} u_{0} \geq 0$ a.e. , but $u_{0}(0) \neq \alpha$, one has $t u^{\varepsilon}$ bounded in $L^{\infty}\left(\mathbb{R}^{+} ; W^{1, \infty}(] 0, L[)\right.$

\section{References}

[1] C. Bardos, A.-Y. Le Roux and J.-C. Nédélec, First order quasilinear equations with boundary conditions, Comm. in PDE, 4 (9), 1017-1034 (1979).

[2] A. Colin, T. Colin, P. Fabrie and D. Monin, Drainage of 3-D foams undergoing coalescence evens, Preprint 1998.

[3] A. Colin, A. Espert, D. Monin, Two mechanisms of coalescence in soap foams : drainage control and homogeneous rupture, submitted to Physical Review Letter.

[4] M. Gisclon, Etude des conditions aux limites pour un système strictement hyperbolique, via l'approximation parabolique. J. Math. Pures Appl. (9) 75 (1996), no. 5, $485-508$.

[5] O. Oleinik, Uniqueness and stability of the generalized solution of the Cauchy problem for a quasilinear equation. Usp. Mat. Nauk., 14 (1959), 165-170; English transl. in Amer. Math. Soc. Transl., Ser.2, 33 (1964), 285-290.

[6] G. Verbist, D. Weaire and A.M. Kraynik, The foam drainage model, J. Phys.: Condens. Matter 8 (1996) 3715-3731.

T.C. colin@math.u-bordeaux.fr

P.F. fabrie@math.u-bordeaux.fr 